\title{
Post-traumatic tension pneumocephalus and cystic angiomatosis of the skull: case report
}

\author{
Haythem H. Osman', Nazik E. Abdullah ${ }^{2 *} \mathbb{0}$, Ghada S. Alhassan' ${ }^{1}$ Tarig A. Nafie ${ }^{3}$, Murtada I. Abdellatief $^{4}$, \\ Ali M. Abdelsatir ${ }^{5}$, Khalid O. Elzein ${ }^{6}$ and Emad I. Ahmed ${ }^{1}$
}

\begin{abstract}
Background: Tension pneumocephalus is an increasing air trapped intracranially. Either spontaneous, post-traumatic or iatrogenic in origin. Cystic angiomatosis is a benign vascular hamartoma of the skeleton, when acquired it is either due to trauma or infection. This is the second report in English literature of post-traumatic delayed tension pneumocephalus with the development of cystic angiomatosis of the skull bone.

Case presentation: A 55-year-old gentlemen, presented with scalp swelling of 6-month duration with history of head trauma 2 years back. The swelling was increasing and associated with progressive walking difficulties and left hearing loss. CT scan and MRI revealed extradural pneumocephalus, parietal and occipital pneumatocele, and multiple lytic bony lesions, left mastoid hyperpneumatization with inner table defect communicating with the extradural space. Diagnosis of delayed extradural tension pneumocephalus was made. Surgical exploration revealed multiple bony defects of parietal, temporal and squamous part of left temporal bones, confirmed extradural pneumocephalus with intact dura. Repair of mastoid defect of $(0.5 \times 0.5 \mathrm{~cm})$, excision of pneumatocele and removal of lytic bones were performed. Defective bone "cribriform-like" was identified at occipital and parietal regions centrally with a defect of nearly $7 \times 7 \mathrm{~cm}$. Future cranioplasty was considered after 6 months. Histology of bony chips and surrounding soft tissues is recognized as cystic angiomatosis.

Conclusions: The present case developed two very rare complications, following trivial head trauma; the first complication was delayed extradural tension pneumocephalus with pneumatocele which presented 2 years after trauma, the origin of air was from a defect of the inner table of the mastoid, the second complication was cystic angiomatosis of the skull bones. Both complications were managed surgically in one operative session as a combined neurosurgery and otolaryngology teams approach.
\end{abstract}

Keywords: Pneumocephalus, Traumatic, Tension, Cystic angiomatosis, Skull

\section{Background}

Pneumocephalus is accumulation of air intracranially, and it is a result of head trauma in nearly $10 \%$ of cases but more commonly iatrogenic as a complication of craniotomy [1]. When it is increasing and associated with neurological symptoms and signs it is termed tension pneumocephalus, which is a rare sequel [2] . It can be of

\footnotetext{
*Correspondence: nazikfad1223@yahoo.co.uk

${ }^{2}$ Department of Otolaryngology, Faculty of Medicine, University

of Khartoum, Qasr Street, P.O. Box 102, Khartoum, Sudan

Full list of author information is available at the end of the article
}

acute onset if developed during the first $72 \mathrm{~h}$ or delayed if developed after $72 \mathrm{~h}$. Increased intracranial pressure with neurological sequels necessitates emergency surgical intervention as the treatment of choice for tension pneumocephalus [3].

Cystic angiomatosis (CA) is a rare benign non progressive bony lesion, classified as skeletal angiomatosis, usually multiple affecting the skull and vertebrae [4], histologically it is characterized by dilated thinwalled capillaries, growing in the marrow space with bone destruction and infiltration into the adjacent soft 
tissues; bone destruction and proliferation of blood vessels are the hallmarks [5].

We report a rare case of head trauma who developed two rare complications: delayed tension pneumocephalus with pneumatocele and cystic angiomatosis. To our knowledge, this is the second report of this condition. Only one case comparable was reported in English literature 30 years ago [6], dissimilar to the present case in two aspects; firstly, being non-tension and early onset of pneumocephalus and secondly, contemporary radiology studies like CT scan and MRI/MRV were performed to demonstrate the lesion for the present case.

\section{Case presentation}

A gentleman of 55 years old presented with progressive bulging of his scalp over 18-month period, left ear hearing impairment, strange feeling in his left ear and walking difficulties. He had a history of trivial head with scalp laceration, 2 years before presentation, for which he was not further investigated, only suture of the cut wound was done. At presentation, he was worried about rapid increase of scalp swelling and unsteady gait over the last week.

Physical and neurological examination revealed large mass under scarred otherwise normal scalp skin, the mass was compressible and when pressed is associated with left ear fullness sensation (see Fig. 1). The patient's gait was unsteady, power of the lower limbs was grade $4+$, and the remaining neurological assessment was normal. Ears examination revealed intact tympanic membranes bilaterally. No hearing tests were performed.

CT scan using a bone algorithm demonstrated a large extradural pneumocephalus, occipital pneumatocele, left mastoid hyperpneumatization, and good aeration with a defect in the inner table of the mastoid that communicated with the extradural space, osteolysis of the occipital and parietal bones extending to the left squamous bones. MRI/MRV revealed huge central occipital and parietal extradural pneumocephalus as the dura was intact and demonstrated a huge pneumatocele, multiple branches and net of vessels draining blood from the mass bulge (pneumatocele) toward the superior sagittal sinuses, no underlying tumor was observed, and slight compression of the temporal lobe of the brain; however, the cerebrum and cerebellum demonstrated normal cortical sulcations. These radiological findings are shown in Fig. 2.

Systemic assessment of the patient included hormonal assays, blood glucose levels, renal and hepatic functions tests and lipid profile, where endocrine and metabolic pathology were excluded.

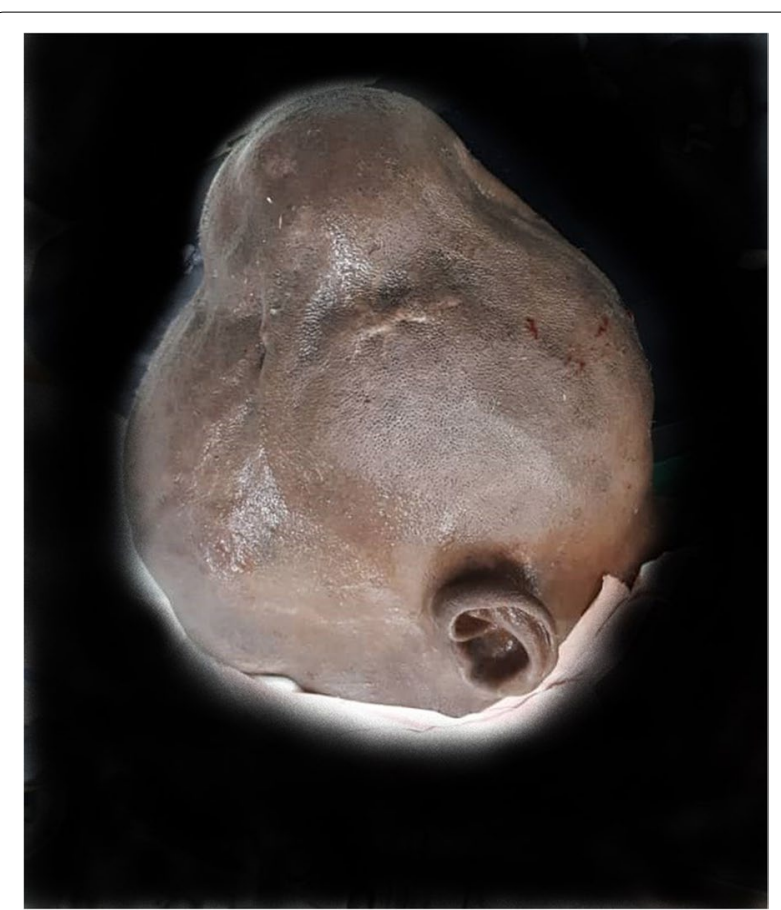

Fig. 1 Scalp swelling and scarred skin

\section{Surgical procedure and findings}

Pneumatocele excision was done by a transverse incision of skin over the bulge, the bulge appeared as transilluminated cyst, beneath skin as in Fig. 3 and Fig. 4 respectively. Cyst-like lesion was collapsed immediately on incision of periosteum, when air emerged.

Defective bone "cribriform-like" was identified at occipital and parietal regions centrally, with soft trabeculae connecting the defective bone to soft tissues above as in Fig. 5A, B. Bone was removed easily by nipping, and irregular edges were removed until healthy bone was recognized with a defect of nearly $7 \times 7 \mathrm{~cm}$. Squamous part of temporal bone was partially involved near the parietal region. Mastoid outer table was intact. Dura was obviously intact hence no cerebro spinal fluid (CSF) leak was observed. The trabeculae were detached and the extradural cavity was irrigated with $1.5 \mathrm{~L}$ of saline.

Cortical mastoidectomy was done via a post-auricular incision, a single circular bony defect at the inner table of sinudural angle was identified $(0.5 \times 0.5 \mathrm{~cm})$, shown in Fig. 5D and was well communicating with the extradural space via a canal-like track, the latter is shown in Fig. 5C, saline irrigation from the mastoid defect was retrieved from craniotomy site. Mastoid defect was sealed watertight by abdominal fat and bone wax. At the end of surgery, pneumatocele was excised and wound was closed as in Fig. 6. 


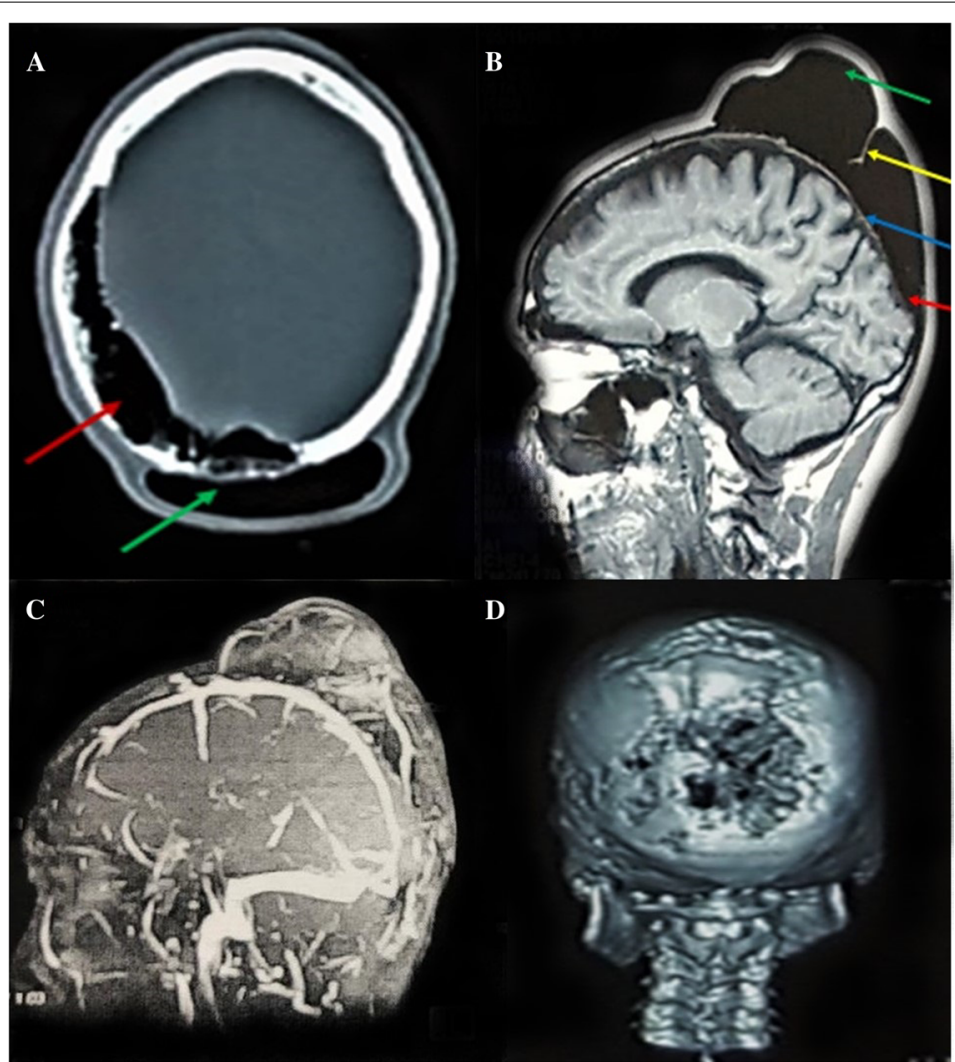

Fig. 2 A CT scan axial cut, bone window, demonstrating, extradural pnemocephalus (red arrow), pneumatocele (green arrow), and lytic skull defects of the occipital bone. B Sagittal MRI T1 weighted, demonstrating huge parietal and occipital pneumocephalus (red arrow) communicating with the pneumatocele (green arrow). Intact dura is demonstrated (blue arrow) and soft tissue trabeculae (yellow arrow) is identified communicating bone defects with the scalp. C MRV study showing multiple branches and net of vessels draining blood from the mass bulge (pneumatocele) toward the superior sinuses. D 3D CT scan of the skull, posterior view showing multiple lytic lesions of the occipital, parietal and left squamous temporal bones

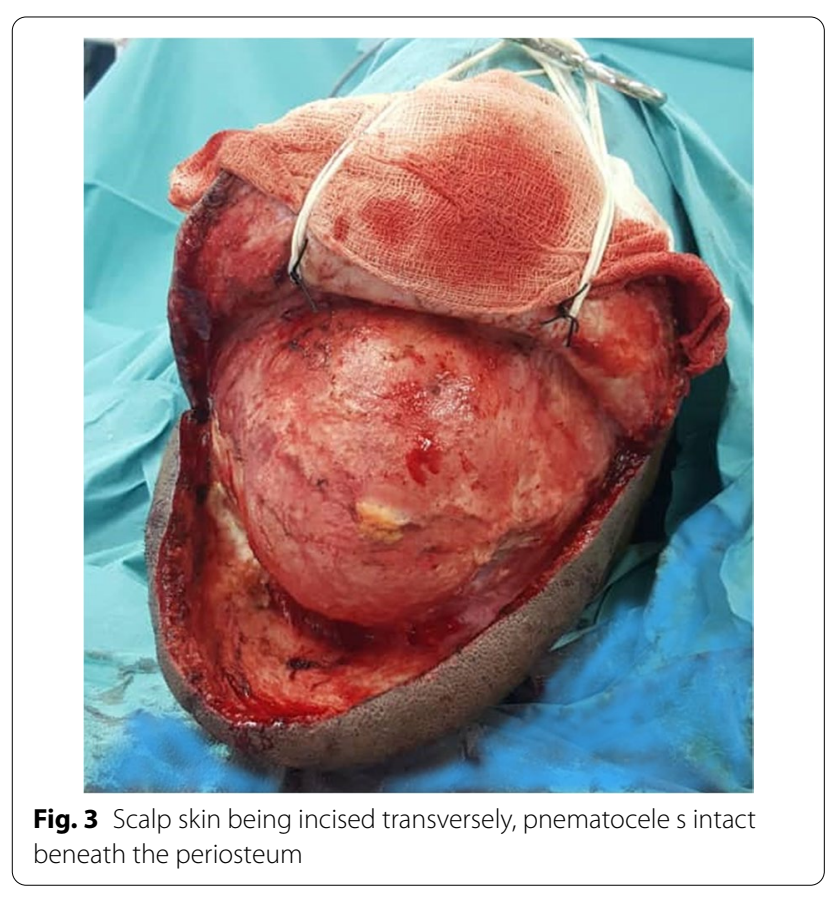

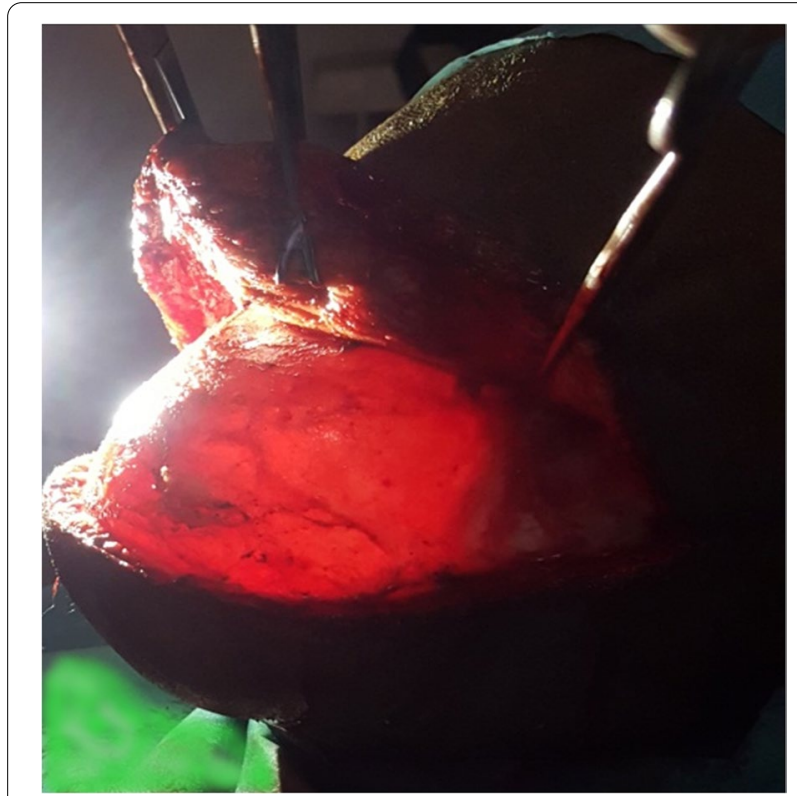

Fig. 4 Operative findings: trans-illuminating cystic lesion of the scalp upon skin incision 


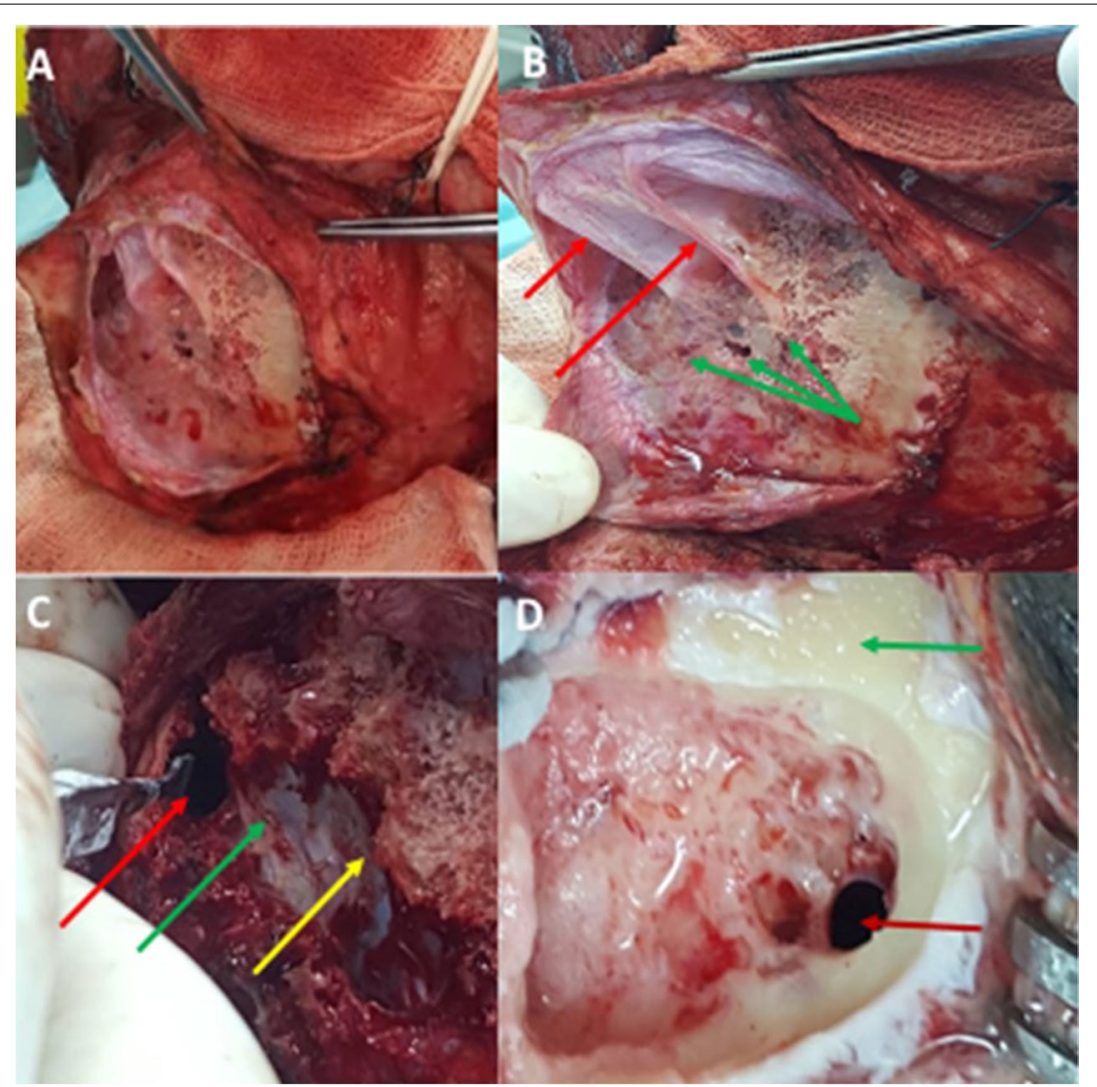

Fig. 5 A, B Operative findings: collapsed pneumatocele after incision of periosteum, trabeculae connecting defective bone (red arrows) with scalp and cribriform like multiple bony defects (green arrows). C Craniotomy with removed lytic skull bones and a dissector being passed from extradural space via a canal "red arrow" to communicate with the mastoid defect in $\mathbf{D}$, green arrow points to the dura and yellow arrow points to the nipped off lytic bone. D Cortical Mastoidectomy of left ear, well circumscribed regular bony defect (red arrow) of inner table of sinudural angle communicating with the extradural space, zygomatic arch is shown by green arrow

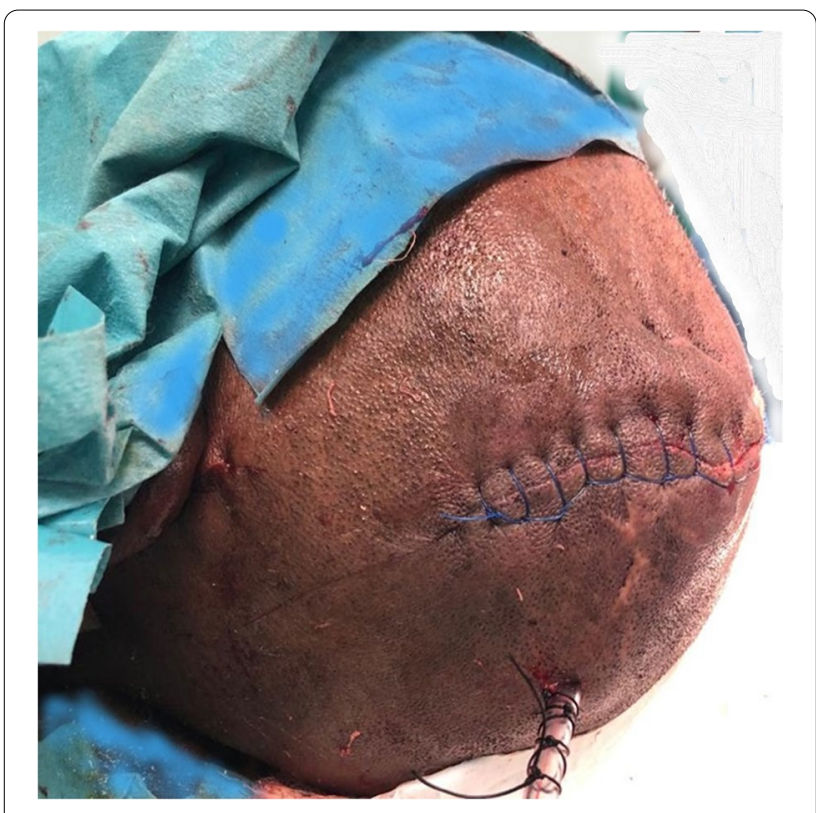

Fig. 6 Scalp incision is closed after pneumatocele excision
Post-operative period was uneventful; the patient was discharged 7 days after surgery and he improved gradually and achieved normal gait, grade 5 lower limbs power and marked hearing improvement 2 weeks thereafter. He is scheduled for a possible second look if cranioplatsy is required after 6 months.

Bony chips from the parietal and occipital region and cuff of surrounding soft tissues were examined histologically, where replacement of bone trabeculae and marrow spaces by multiple distended blood vessels was the principal feature, with diffuse network of small and large, empty, anastomosing, vascular channels. The endothelial lining of the vascular channels was flat and normal in appearance, with no mitosis or tufting of the endothelial cells. Extensive sampling of the lesion did not demonstrate any of the features of angiosarcoma.

The final pathologic diagnosis was cystic angiomatosis (diffuse hemangioma) as shown in Fig. 7, associated with extradural pneumocephalus. 


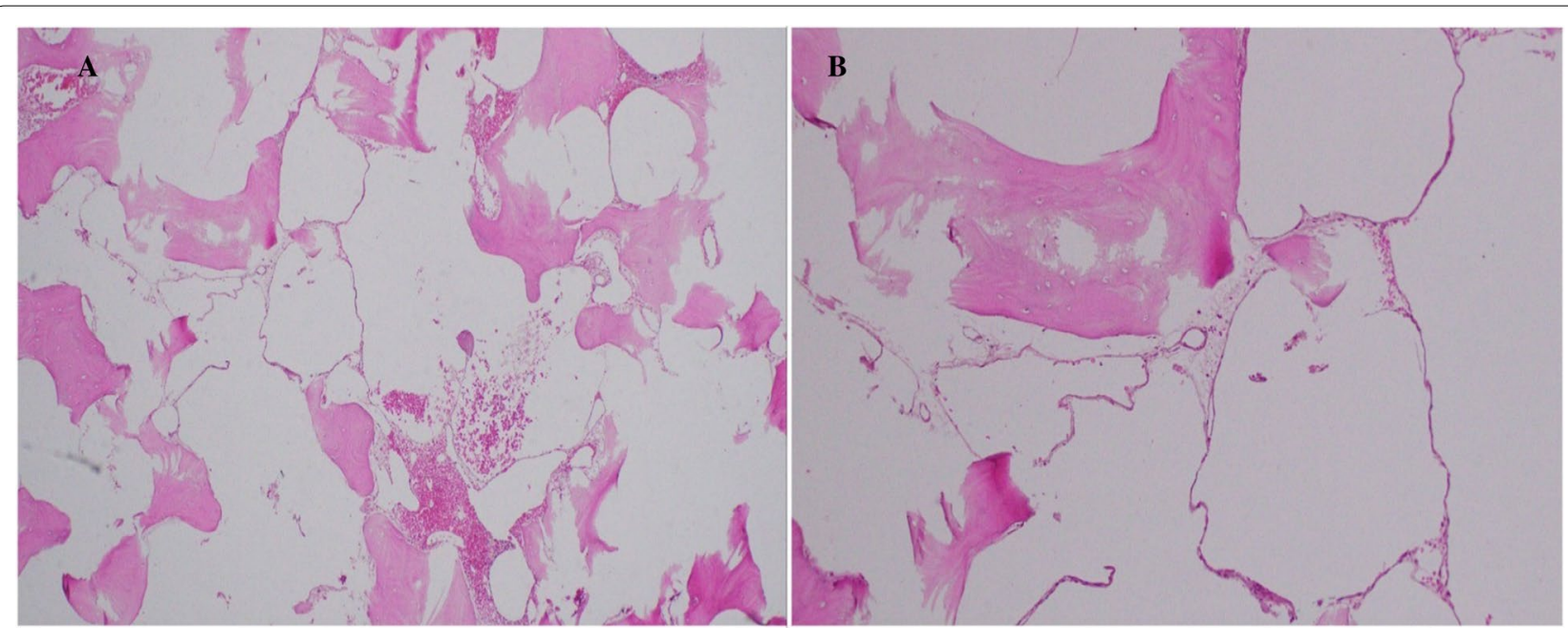

Fig. 7 A Microscopy of hematoxylin and eosin, skull mass shows replacement of bone trabeculae and marrow space by multiple distended blood vessels. B Microscopy of high magnification shows multiple blood vessels with red blood cells and flat endothelial lining (arrow)

\section{Conclusions}

In the present case, air was trapped from the mastoid air cells in the extradural space via a defect in the inner table of the mastoid, in a one-way valve mechanism. The mastoid defect is most likely due to fracture from previous trauma dated 2 years back. Trivial trauma can lead to fracture of a high pneumatized mastoid, because this can happen spontaneously as reported by Santiago et al. [7], delay of presentation in the present case is suggested to be due to edematous tissues at the site of fracture that sealed temporary a mastoid defect.

Pneumatocele developed in the present case as a result of multiple occipital and parietal bony defects that permit air to escape from extradural space, despite this there was no relief of pneumocephalus as the patient remained symptomatic with walking difficulties indicating that this is a tension type of pneumocephalus.

Lytic bony lesions of the parietal and occipital bones are suggested to be due to osteolysis-associated angiomatosis [4], and/or focal osteolysis caused by trauma, it is reported that trauma induces periosteal nonspecific inflammatory reaction with consequent bone resorption [8].

Cystic angiomatosis is either congenital or acquired, when acquired it is caused by infections or trauma [9]. When limited to the skull and no visceral involvement it carries good prognosis [6]. Treatment of skull cystic angiomatosis is usually surgical excision, which is not always easy, e.g., when present over the transverse sinus it is difficult to be excised without significant morbidity [10]. A novel procedure is advented recently with promising results, by Guilherme et al., to treat difficult occipital skull lesions directly overlying the transverse sinus, and the procedure is done by performing a small, partial-thickness craniectomy and alcohol sclerotherapy in a combined neurosurgery-neuroendovascular approach [10].

\section{Abbreviations \\ CT: Computed tomography; MRI: Magnetic resonance image; CA: Cystic angio- matosis; MRV: Magnetic resonance venography; CSF: Cerebrospinal fluid.}

\section{Acknowledgements}

We would like to acknowledge the theater staff of The National Ribat University Hospital for their dedication and efforts in the surgical management of the patient.

\section{Authors' contributions}

All authors have read and approved the manuscript. NEA done the conception, design, acquisition of data, analysis of data, interpretation of data and revising manuscript. $\mathrm{HHO}$ done the conception, design, acquisition of data, analysis of data, interpretation of data and drafting manuscript. GSA done the design, acquisition of data, analysis of data, interpretation of data and drafting manuscript. TAN done design, analysis of data, interpretation of data and revising manuscript. MIA done the conception, acquisition of data, analysis of data and interpretation of data. AMA done the design, acquisition of data, analysis of data and interpretation of data. KOE done the acquisition of data and revising manuscript. ElA done acquisition of data and revising manuscript.

\section{Funding}

No financial support was provided to this work.

\section{Availability of data and materials}

Authors can confirm that all relevant data are included in the article and/or its supplementary information files. 


\section{Declarations}

\section{Ethics approval and consent to participate}

Reporting of this case was approved by the Research Ethical Committee of the National Ribat University Hospital, Khartoum, Sudan. No reference number was allocated. Written informed consent for participation was obtained from the patient.

\section{Consent for publication}

Written informed consent for publication of the clinical details and clinical images was obtained from the patient. The patient agreed upon the fact that the text and any pictures published in the article will be freely available on the internet and may be seen by the general public. The pictures and text may also appear on other websites or in print, may be translated into other languages or used for commercial purposes. A copy of the consent form is available for review by the Editor of this journal.

\section{Competing interests}

The authors declare that they have no competing interests.

\section{Author details}

'The National Ribat University, Neurosurgery and Spine Center, Ribat University Hospital, Khartoum, Sudan. ${ }^{2}$ Department of Otolaryngology, Faculty of Medicine, University of Khartoum, Qasr Street, P.O. Box 102, Khartoum, Sudan. ${ }^{3}$ Otology Department, ENT-Khartoum Teaching Hospital, Khartoum, Sudan. ${ }^{4}$ Department of Radiology, Ribat University Hospital, Khartoum, Sudan. ${ }^{5}$ Histo Center, Khartoum, Sudan. ${ }^{6}$ Department of Otolaryngology, The National Ribat University Hospital, Khartoum, Sudan

Received: 29 May 2021 Accepted: 12 November 2021

Published online: 04 December 2021

\section{References}

1. Komolafe EO (2010) Tension pneumocephalus: a rare but treatable cause of rapid neurological deterioration in traumatic brain injury: a case report. Afr J Med Sci 29:88-91
2. Leong KM, Vijayananthan A, Sia SF, Waran V (2008) Pneumocephalus: an uncommon finding in trauma. Med J Malaysia 63:256-258

3. Kwon J, Rha HK, Park HK, Chough CK, Joo Wl, Cho SH, Wonmo G, Moon W Han J (2017) Proper management of posttraumatic tension pneumocephalus. Korean J Neurotrauma 13(2):158-161

4. Malik R, Malik R, Tandon S, Tandon P (2008) Skeletal angiomatosis - rare cause of bone destruction: a case report with review of literature. Indian J Pathol Microbiol 51:515-518

5. Cobby M, Watt I (2003) Tumors and tumor like conditions of bone. In: David Sutton editor. Text Book of Radiology and Imaging, Churchill Living Stone p, pp 1287-1320

6. Morshed A, Mohit P (1990) Cystic angiomatosis of the skull presenting with extradural pneumocephalus case report. J Neurosurg 72:968-970

7. Abbati SG, Torino RR (2012 Mar) Spontaneous intraparenchymal otogenic pneumocephalus: a case report and review of literature. Surg Neurol Int. https://doi.org/10.4103/2152-7806.93861

8. Hornig GW, Beatty RM (1990 Mar) Osteolytic skull lesions secondary to trauma. Report of two cases. J Neurosurg 72(3):506-508

9. Kirma C, Izgi A, Yakut C, Guler M, Can M, Zemheri E (2006) Primary left ventricular angiomatosis. Int Heart J 47:469-474

10. (2020 Apr) Cystic angiomatosis skull lesion obliteration with neuroendovascular sclerotherapy as a unique treatment: case report. J Neurosurg Pediatr. Guilherme B. F. Porto, Cynthia T. Welsh, M. Imran Chaudry, Ramin Eskandari. https://doi.org/10.3171/2020.3.PEDS2045

\section{Publisher's Note}

Springer Nature remains neutral with regard to jurisdictional claims in published maps and institutional affiliations. 